\title{
CHARACTERIZATIONS OF GENERALIZED HERMITE AND SIEVED ULTRASPHERICAL POLYNOMIALS
}

\author{
HOLGER DETTE
}

\begin{abstract}
A new characterization of the generalized Hermite polynomials and of the orthogonal polynomials with respect to the measure $|x|^{\gamma}\left(1-x^{2}\right)^{1 / 2} d x$ is derived which is based on a "reversing property" of the coefficients in the corresponding recurrence formulas and does not use the representation in terms of Laguerre and Jacobi polynomials. A similar characterization can be obtained for a generalization of the sieved ultraspherical polynomials of the first and second kind. These results are applied in order to determine the asymptotic limit distribution for the zeros when the degree and the parameters tend to infinity with the same order.
\end{abstract}

\section{INTRODUCTION}

Consider the generalized Hermite polynomials $H_{n}^{(\gamma)}(x)$ orthogonal with respect to the measure $|x|^{\gamma} \exp \left(-x^{2}\right) d x(\gamma>-1)$. A characterization of these polynomials can easily be obtained from a characterization of the Laguerre polynomials (see e.g. Al-Salam [3] or Chihara [8, p. 142]) and the well known relations between Laguerre- and Hermite polynomials

$$
H_{2 n}^{(\gamma)}(x)=(-1)^{n} 2^{2 n} n ! L_{n}^{(\gamma / 2-1 / 2)}\left(x^{2}\right),
$$

$$
H_{2 n+1}^{(\gamma)}(x)=(-1)^{n} 2^{2 n+1} n ! x L_{n}^{(\gamma / 2+1 / 2)}\left(x^{2}\right)
$$

(see $[8$, p. 156]). In this paper we present two new characterizing properties of the generalized Hermite polynomials which are based on a "reversing property" of the coefficients in the corresponding three term recurrence relations and do not use explicitly the relation (1). Similar results can be derived for orthogonal polynomials on a compact interval, say $[-1,1]$. Here the analogue of the generalized Hermite polynomials (with respect to the new characterizations) are the orthogonal polynomials on the interval $[-1,1]$ with respect to the measure $|x|^{\gamma}\left(1-x^{2}\right)^{1 / 2} d x$ (see $[8$, p. 156]) which satisfy a similar "reversing property". While the orthogonal polynomials with respect to the measures $|x|^{\gamma} \exp \left(-x^{2}\right) d x$ and $|x|^{\gamma}\left(1-x^{2}\right)^{1 / 2} d x$ are discussed in Section 2, Section 3 investigates analogous characterizations for some "relatively new" systems of orthogonal polynomials, namely the sieved random walk polynomials introduced by Charris and Ismail [5] (see also Ismail [12],

Received by the editors June 5, 1994 and, in revised form, January 10, 1995.

1991 Mathematics Subject Classification. Primary 33C45.

Key words and phrases. Generalized Hermite polynomials, sieved ultraspherical polynomials, Stieltjes transform, continued fractions, asymptotic zero distribution. 
Charris and Ismail $[6,7]$ and Geronimo and VanAssche [11]). We give a characterization for a generalization of the sieved ultraspherical polynomials of the first and second kind, $C_{n}^{(\alpha, \gamma)}(x, k), B_{n}^{(\alpha, \gamma)}(x, k)$, which were introduced by Askey [4] as a limit of the $q$-Wilson polynomials and are orthogonal on the interval $[-1,1]$ with respect to the measures with densities

$$
w_{1}(x, \alpha, \gamma)=\left(1-x^{2}\right)^{\alpha-1 / 2}\left|U_{k-1}(x)\right|^{2 \alpha}\left|T_{k}(x)\right|^{\gamma}
$$

and

$$
w_{2}(x, \alpha, \gamma)=\left(1-x^{2}\right)^{\alpha+1 / 2}\left|U_{k-1}(x)\right|^{2 \alpha}\left|T_{k}(x)\right|^{\gamma}
$$

(here $k \in \mathbb{N}$ is a fixed integer and $T_{k}(x)$ and $U_{k-1}(x)$ denote the Chebyshev polynomials of the first and second kind). In the case $\gamma=0$ these polynomials were obtained by Al-Salam, Allaway and Askey [2] as a limit from the $q$-ultraspherical polynomials of Rogers [16].

Finally the results of Sections 2 and 3 are applied in Section 4 in order to derive the asymptotic zero distribution of the polynomials $H_{n}^{(\gamma)}(x), C_{n}^{(\alpha, \gamma)}(x, k)$ and $B_{n}^{(\alpha, \gamma)}(x, k)$ when the degree and parameters tend to infinity with the same order. For the generalized Hermite polynomials we thus obtain an alternative proof of a recent result in Gawronski [10] while the limit distribution for the zeros of the generalized sieved ultraspherical polynomials of the first and second kind is a generalization of the measure for which the sieved Chebyshev polynomials of the first kind are orthogonal with respect to (see [12]).

Our motivation for investigating and characterizing polynomials of this type stems from the theory of optimal experimental design, although there are no direct applications of the results of the present paper in this field. In many cases optimal designs for polynomial regression models can be shown to be supported at the zeros of certain (classical) orthogonal polynomials. While for observations with constant variance the solution of the design problem is related to the Legendre polynomials, observations with variances reversely proportional to the Beta, Gamma, and Gaussian density functions yield solutions of the design problem which are related to the Jacobi- Laguerre- and Hermite polynomials. For more details about the application of orthogonal polynomials in the theory of optimal design the reader is referred to the work of Karlin and Studden [13, p. 333] and Lau and Studden [14].

\section{Generalized Hermite polynomials AND THE WEIGHT FUNCTION $|x|^{\gamma}\left(1-x^{2}\right)^{\alpha-1 / 2}$}

Let $\mu$ denote a probability measure on the real line $(-\infty, \infty)$ with all moments existing. The Stieltjes transform of $\mu$ has the continued fraction expansion

$$
\Phi(z)=\int_{-\infty}^{\infty} \frac{d \mu(x)}{z-x}=\frac{1 \mid}{\mid z-b_{1}}-\frac{a_{1} \mid}{\mid z-b_{2}}-\frac{a_{2} \mid}{\mid z-b_{3}}-\ldots
$$

where the quantities $a_{i} \geq 0, b_{i} \in \mathbb{R}(i \geq 1)$ can be expressed in terms of the ordinary moments of $\mu$ (see Perron [15, p. 193], or Wall [19, p. 258]). In the following we consider the $n$th "truncated" continued fraction of (2.1) (i.e. we put $a_{n+1}=0$ ) and its corresponding probability measure $\mu_{n}$, that is

$$
\Phi_{n}(z)=\int_{-\infty}^{\infty} \frac{d \mu_{n}(x)}{z-x}=\frac{1 \mid}{\mid z-b_{1}}-\frac{a_{1} \mid}{\mid z-b_{2}}-\ldots-\frac{a_{n} \mid}{\mid z-b_{n+1}} .
$$

It is well known that $\mu_{n}$ has finite support given by the zeros of the polynomial in the denominator of (2.2). Moreover, it can be shown (see Dette and Studden [9, p. 
4]) that the measure $\mu_{n}^{R}$ with Stieltjes transform corresponding to the "reversed" continued fraction

$$
\Phi_{n}^{R}(z)=\int_{-\infty}^{\infty} \frac{d \mu_{n}^{R}(x)}{z-x}=\frac{1}{\mid z-b_{n+1}}-\frac{a_{n} \mid}{\mid z-b_{n}}-\ldots-\frac{a_{1} \mid}{\mid z-b_{1}}
$$

has the same support points as $\mu_{n}$. In the following we are interested in measures for which this "reversed" measure is "nearly" a uniform distribution on its support points. More precisely, we ask for all probability measures on $(-\infty, \infty)$ with the property

$$
\left\{\begin{array}{l}
- \text { If } n=2 m-1 \text { is odd }(n \in \mathbb{N}), \text { then } \mu_{n}^{R} \text { has equal masses at all } \\
n \text { support points } \\
- \text { If } n=2 m \text { is even }(n \in \mathbb{N}), \text { then } \mu_{n}^{R} \text { has equal masses at } n \text { support } \\
\text { points and positive mass at a point } x_{0} \text { (independent of } n \text { ). }
\end{array}\right.
$$

The following theorem shows that there is exactly one probability measure with the property (2.4) (up to a linear transformation), namely the measure with density proportional to the weight function of the generalized Hermite polynomials.

Theorem 2.1. The generalized Hermite polynomials $H_{n}^{(\gamma)}(x)$ orthogonal with respect to the measure $d \xi_{\gamma}(x)=|x|^{\gamma} \exp \left(-x^{2}\right) d x(\gamma>-1)$ can be characterized as the unique (up to a linear transformation) orthogonal polynomials on $(-\infty, \infty)$ whose corresponding probability measure satisfies (2.4) for all $n \in \mathbb{N}$. Moreover, for all $m \in \mathbb{N}$ the weight of $\xi_{\gamma, 2 m}^{R}$ at the point 0 is $\gamma+1$ times bigger than the (equal) weight of $\xi_{\gamma, 2 m}^{R}$ at the remaining $2 m$ support points.

Proof. In a first step we show that the probability measure $\xi_{\gamma}$ with density proportional to the function $|x|^{\gamma} \exp \left(-x^{2}\right)$ satifies (2.4). To this end let $H_{n}^{(\gamma)}(x)$ denote the $n$th generalized Hermite polynomial and $K_{n}^{(\gamma)}(x)$ its monic form. From [8, p. 157] we have the recursive relation $\left(K_{-1}^{(\gamma)}(x)=0, K_{0}^{(\gamma)}(x)=1\right)$

$$
K_{n+1}^{(\gamma)}(x)=x K_{n}^{(\gamma)}(x)-\hat{a}_{n} K_{n-1}^{(\gamma)}(x)
$$

where

$$
\hat{a}_{n}= \begin{cases}\frac{n}{2} & \text { if } n \text { is even, } \\ \frac{n+\gamma}{2} & \text { if } n \text { is odd } .\end{cases}
$$

Consequently the quantities $b_{j}$ in the continued fraction expansion of the Stieltjes transform of $\xi_{\gamma}$ in (2.1) satisfy $b_{j}=0$ while the "numerators" $a_{j}$ are given by (2.6). Thus we obtain from (2.3)

$$
\Phi_{n}^{R}(z)=\int_{-\infty}^{\infty} \frac{d \xi_{\gamma, n}^{R}(x)}{z-x}=\frac{1 \mid}{\mid z}-\frac{\hat{a}_{n} \mid}{\mid z}-\ldots-\frac{\hat{a}_{1} \mid}{\mid z} .
$$


Now let $n=2 m\left(m \in \mathbb{N}_{0}\right)$, then it follows from $K_{j}^{(\gamma)}(z)=2^{-j} H_{j}^{(\gamma)}(z),(2.5),(2.6)$ and formula $(2.47)$ in $[8$, p. 157] that

$$
\begin{aligned}
\frac{d}{d z} K_{2 m+1}^{(\gamma)}(z) & =(2 m+1) K_{2 m}^{(\gamma)}(z)+\frac{m \gamma}{z} K_{2 m-1}^{(\gamma)}(z) \\
& =(2 m+1+\gamma) K_{2 m}^{(\gamma)}(z)-\frac{\gamma}{z} K_{2 m+1}^{(\gamma)}(z)
\end{aligned}
$$

and formula (2.7) yields that the support of $\xi_{\gamma, 2 m}^{R}$ is given by the zeros of $K_{2 m+1}^{(\gamma)}(z)$. Whenever $K_{2 m+1}^{(\gamma)}\left(z_{0}\right)=0$ and $z_{0} \neq 0$ we have from $(2.8)$

$$
\xi_{\gamma, 2 m}^{R}\left(z_{0}\right)=\frac{K_{2 m}^{(\gamma)}\left(z_{0}\right)}{\left.\frac{d}{d z} K_{2 m+1}^{(\gamma)}(z)\right|_{z=z_{0}}}=\frac{1}{2 m+1+\gamma}
$$

Consequently, $\xi_{\gamma, 2 m}^{R}$ has equal masses $\frac{1}{2 m+1+\gamma}$ at the zeros of $K_{2 m+1}^{(\gamma)}(z)$ which are different from 0 and mass $\frac{\gamma+1}{2 m+1+\gamma}$ at the point 0 . In the case $n=2 m-1$ we obtain by a similar reasoning that

$$
\frac{d}{d z} K_{2 m}^{(\gamma)}(z)=2 m K_{2 m-1}^{(\gamma)}(z)
$$

which implies for all $z_{0} \in \operatorname{supp}\left(\xi_{\gamma, 2 m-1}^{R}\right)=\left\{z \mid K_{2 m}^{(\gamma)}(z)=0\right\}$

$$
\xi_{\gamma, 2 m-1}^{R}\left(z_{0}\right)=\frac{K_{2 m-1}^{(\gamma)}\left(z_{0}\right)}{\left.\frac{d}{d z} K_{2 m}^{(\gamma)}(z)\right|_{z=z_{0}}}=\frac{1}{2 m}
$$

This proves that the probability measure with density proportional to $|x|^{\gamma} \exp \left(-x^{2}\right)$ satisfies the reversing property (2.4).

In a second step we now show that there is no other probability measure with this property. If $n=2 m-1$, then it follows from [9, p. 16] that the property (2.4) implies for $m \geq 1$

$$
(2 m-1) \sum_{i=1}^{2 m} b_{i}=2 m \sum_{i=1}^{2 m-1} b_{i}
$$

$$
(2 m-2)\left[\sum_{1 \leq i<j \leq 2 m} b_{i} b_{j}-\sum_{i=1}^{2 m-1} a_{i}\right]=2 m\left[\sum_{1 \leq i<j \leq 2 m-1} b_{i} b_{j}-\sum_{i=1}^{2 m-2} a_{i}\right] .
$$

If $n=2 m$ we denote by $x_{0}, x_{1}, \ldots, x_{2 m}$ the support points of a reversed measure $\mu_{2 m}^{R}$ satisfying (2.4) (note that $x_{1}, \ldots, x_{2 m}$ depend on $2 m$ and $x_{0}$ is independent 
of $2 m$ ) and obtain for the Stieltjes transform for some $\gamma>-1$

$$
\begin{aligned}
\Phi_{2 m}^{R}(z)= & \frac{1}{2 m+1+\gamma} \sum_{j=1}^{2 m} \frac{1}{z-x_{j}}+\frac{\gamma+1}{2 m+1+\gamma} \frac{1}{z-x_{0}} \\
& \frac{\prod_{j=0}^{2 m}\left(z-x_{j}\right)^{-1}}{2 m+1+\gamma}\left[\begin{array}{c}
(2 m+1+\gamma) z^{2 m}-\left\{(2 m+\gamma) \sum_{i=0}^{2 m} x_{i}-\gamma x_{0}\right\} z^{2 m-1} \\
\left.\quad+\left\{(2 m-1+\gamma) \sum_{0 \leq i<j \leq 2 m} x_{i} x_{j}-\gamma x_{0} \sum_{i=1}^{2 m} x_{i}\right\} z^{2 m-2}-\ldots\right]
\end{array}\right.
\end{aligned}
$$

On the other hand the coefficient of $z^{2 m-1}$ and $z^{2 m-2}$ of the polynomial in the numerator of $\Phi_{2 m}^{R}(z)$ in $(2.3)$ can be written as (see [15, p. 7])

$$
-\sum_{i=1}^{2 m} b_{i}, \quad \sum_{1 \leq i<j \leq 2 m} b_{i} b_{j}-\sum_{i=1}^{2 m-1} a_{i}
$$

respectively, while it is easy to see that

$$
\sum_{i=0}^{2 m} x_{i}=\sum_{i=1}^{2 m+1} b_{i}, \quad \sum_{0 \leq i<j \leq 2 m} x_{i} x_{j}=\sum_{1 \leq i<j \leq 2 m+1} b_{i} b_{j}-\sum_{i=1}^{2 m} a_{i} .
$$

Thus the property (2.4) (in the case $n=2 m, m \geq 0$ ) implies the equations

$$
\begin{gathered}
(2 m+\gamma) \sum_{i=1}^{2 m+1} b_{i}-\gamma x_{0}=(2 m+1+\gamma) \sum_{i=1}^{2 m} b_{i} \\
(2 m-1+\gamma)\left[\sum_{1 \leq i<j \leq 2 m+1} b_{i} b_{j}-\sum_{i=1}^{2 m} a_{i}\right]-\gamma x_{0}\left[\sum_{i=1}^{2 m+1} b_{i}-x_{0}\right] \\
=(2 m+1+\gamma)\left[\sum_{1 \leq i<j \leq 2 m} b_{i} b_{j}-\sum_{i=1}^{2 m-1} a_{i}\right]
\end{gathered}
$$

which can easily be rewritten as

$$
b_{2 m+1}=\frac{1}{2 m+\gamma}\left[\sum_{i=1}^{2 m} b_{i}+\gamma x_{0}\right]
$$

$$
\begin{aligned}
(2 m-1+\gamma) a_{2 m} & =2 \sum_{i=1}^{2 m-1} a_{i}-\gamma x_{0}\left[\sum_{i=1}^{2 m+1} b_{i}-x_{0}\right]-2 \sum_{1 \leq i<j \leq 2 m} b_{i} b_{j} \\
& +(2 m-1+\gamma) b_{2 m+1} \sum_{i=1}^{2 m} b_{i}
\end{aligned}
$$


$(m \geq 0)$. From the first equations in (2.9) and (2.10) it follows that $b_{j}=x_{0}$ for all $j \in \mathbb{N}$ while the second equations reduce to

$$
a_{2 m-1}=\frac{2}{2 m-2} \sum_{j=1}^{2 m-2} a_{j}, \quad m \geq 2,
$$

$$
a_{2 m}=\frac{2}{2 m-1+\gamma} \sum_{j=1}^{2 m-1} a_{j}, \quad m \geq 1 .
$$

An induction argument now proves that all solutions of (2.12) are of the form

$$
a_{j}=\left\{\begin{array}{ll}
\frac{j}{2} c^{-2} & \text { if } j \text { is even } \\
\frac{j+\gamma}{2} c^{-2} & \text { if } j \text { is odd }
\end{array} \quad(j \in \mathbb{N}, c>0)\right.
$$

and by the discussion in the first part of the proof the measure $\mu$ corresponding to this sequence has the density proportional to $\left|c\left(x-x_{0}\right)\right|^{\gamma} \exp \left(-\left|c\left(x-x_{0}\right)\right|^{2}\right)$. This completes the proof of Theorem 2.1

For $\gamma=0$ Theorem 2.1 reduces to the characterization of the Hermite polynomials given in [9]. In this case the measure $\xi_{\gamma, n}^{R}$ puts equal masses at its support points or all $n \in \mathbb{N}$. The following result provides a slightly different characterization of the generalized Hermite polynomials in the class of all symmetric polynomials.

Theorem 2.2. The generalized Hermite polynomials $H_{n}^{(\gamma)}(x)$ orthogonal with respect to the measure $d \xi_{\gamma}(x)=|x|^{\gamma} \exp \left(-x^{2}\right) d x(\gamma>-1)$ can be characterized as the unique (up to a scaling factor) symmetric orthogonal polynomials whose corresponding probability measure $\mu$ satisfies for all $m \in \mathbb{N}$

$$
\begin{aligned}
& \mu_{2 m}^{R} \text { has equal masses at all support points which are } \\
& \text { different from zero. }
\end{aligned}
$$

Moreover, for all $m \in \mathbb{N}$ the weight of $\xi_{\gamma, 2 m}^{R}$ at the point 0 is $\gamma+1$ times bigger than the (equal) weight of $\xi_{\gamma, 2 m}^{R}$ at the remaining $2 m$ support points.

Proof. The proof is similar to that of Theorem 2.1 and we only sketch the main steps. By the discussion in the first part of the proof of the previous theorem it follows that the measure $\xi_{\gamma}$ with density proportional to $|x|^{\gamma} \exp \left(-x^{2}\right)$ satisfies (2.13). Assume now that $\mu$ is a symmetric measure such that (2.13) holds for all $m \in \mathbb{N}$. By the symmetry of $\mu$ we have $b_{i}=0(i \in \mathbb{N})$ and for some $\gamma>-1$

$$
\begin{aligned}
& \Phi_{2 m}^{R}(z)= \int_{-\infty}^{\infty} \frac{d \mu_{2 m}^{R}(x)}{z-x}=\frac{1 \mid}{\mid z}-\frac{a_{2 m} \mid}{\mid z}-\ldots-\frac{a_{1} \mid}{\mid z}=z^{-1} \prod_{j=1}^{2 m}\left(z-x_{j}\right)^{-1} \\
& \times {\left[z^{2 m}-z^{2 m-2} \frac{2 m-1+\gamma}{2 m+1+\gamma} \sum_{1 \leq i<j \leq 2 m} x_{i} x_{j}+z^{2 m-4} \frac{2 m-3+\gamma}{2 m+1+\gamma}\right.} \\
&\left.\times \sum_{1 \leq i<j<k<l \leq 2 m} x_{i} x_{j} x_{k} x_{l} \ldots\right]
\end{aligned}
$$


where $x_{0}=0, x_{1}, \ldots, x_{2 m}$ denote the $2 m+1$ support points of the (symmetric) measure $\mu_{2 m}^{R}$. Comparing coefficients of the two representations for the polynomial in the denominator of this continued fraction (similarly as in the second part of the proof of Theorem 2.1) now yields the equations

$$
a_{2 m}=\frac{2}{2 m-1+\gamma} \sum_{i=1}^{2 m-1} a_{i}, \quad m \geq 1,
$$

$$
a_{2 m} \sum_{i=1}^{2 m-2} a_{i}=\frac{4}{2 m-3+\gamma} \sum_{1 \leq i<j \leq 2 m-2} a_{i} a_{j+1}, \quad m \geq 2 .
$$

A tedious calculation shows that $(2.14)$ is equivalent to $\left(a_{0}=0\right)$

$$
\begin{array}{ll}
a_{2 m}=\frac{2}{2 m-1+\gamma} a_{2 m-1}+a_{2 m-2}, & m \geq 1, \\
a_{2 m}=\frac{4}{2 m-1+\gamma} a_{2 m-1}+a_{2 m-4}, & m \geq 2
\end{array}
$$

and an induction argument proves that the (unique) solution of this system is given by (2.12). This completes the proof of the theorem.

In the remaining part of this section we will concentrate on symmetric distributions on the interval $[-1,1]$ for which similar characterizations can be derived. For the sake of brevity we only consider a characterization of the type (2.13). Let $\mu$ denote a symmetric probability measure on the interval $[-1,1]$ with Stieltjes transform

$$
\Phi(z)=\int_{-1}^{1} \frac{d \mu(x)}{z-x}=\frac{1 \mid}{\mid z}-\frac{p_{2} \mid}{\mid z}-\frac{q_{2} p_{4} \mid}{\mid z}-\frac{q_{4} p_{6} \mid}{\mid z}-\ldots
$$

where $p_{2 i} \in[0,1], q_{2 i}=1-p_{2 i}(i \in \mathbb{N}), q_{0}=1$ and $\left(q_{2 i-2} p_{2 i}\right)_{i \in \mathbb{N}}$ is the minimal chain sequence in the recursive relation of the monic orthogonal polynomials with respect to the measure $d \mu(x)$. If $(2.15)$ holds for a symmetric probability measure $\mu$ (on the interval $[-1,1])$ we write that " $\mu$ corresponds to the sequence $\left(p_{2}, p_{4}, p_{6}, \ldots\right)$ ". If $\mu_{n}$ denotes the (finite) measure corresponding to the "terminated" continued fraction

$$
\Phi_{n}(z)=\int_{-1}^{1} \frac{d \mu_{n}(x)}{z-x}=\frac{1 \mid}{\mid z}-\frac{p_{2} \mid}{\mid z}-\frac{q_{2} p_{4} \mid}{\mid z}-\ldots-\frac{q_{2 n-2} p_{2 n} \mid}{z}
$$

we write that " $\mu_{n}$ corresponds to the sequence $\left(p_{2}, \ldots, p_{2 n}, 0\right)$ " and denote by $\mu_{n}^{R}$ the measure corresponding to the "reversed" sequence $\left(p_{2 n}, \ldots, p_{2}, 0\right)$. The Stieltjes transform of $\mu_{n}^{R}$ is given by

$$
\Phi_{n}^{R}(z)=\int_{-1}^{1} \frac{d \mu_{n}^{R}(x)}{z-x}=\frac{1 \mid}{\mid z}-\frac{p_{2 n} \mid}{\mid z}-\frac{q_{2 n} p_{2 n-2} \mid}{\mid z}-\ldots-\frac{q_{4} p_{2} \mid}{\mid z}
$$

and it is known that $\mu_{n}$ and $\mu_{n}^{R}$ have the same support points (see [9]).

Throughout this paper we define $C_{n}^{(\alpha, \gamma)}(x)$ as the polynomials orthogonal on the interval $[-1,1]$ with respect to the measure $|x|^{\gamma}\left(1-x^{2}\right)^{\alpha-1 / 2} d x$ (without specifying any normalization). The following result is the analogue of Theorem 2.2 on the 
interval $[-1,1]$ and can be proved by similar arguments as given in the proof of Theorem 2.2 .

Theorem 2.3. The polynomials $C_{n}^{(\alpha, \gamma)}(x)$ orthogonal with respect to the measure $d \xi_{\alpha, \gamma}^{*}(x)=|x|^{\gamma}\left(1-x^{2}\right)^{\alpha-1 / 2} d x(\alpha>-1 / 2, \gamma>-1)$ can be characterized as the unique symmetric orthogonal polynomials whose corresponding probability measure $\mu$ satisfies for all $m \in \mathbb{N}$

$$
\mu_{2 m}^{R} \text { has equal masses at all support points different from } 0 .
$$

Moreover, for all $m \in \mathbb{N}$ the weight of $\xi_{\alpha, \gamma, 2 m}^{R^{*}}$ at the point 0 is $\gamma+1$ times bigger than the (equal) weight of $\xi_{\alpha, \gamma, 2 m}^{R^{*}}$ at the remaining $2 m$ support points.

Proof. We will only show that the probability measure $\xi_{\alpha, \gamma}^{*}$ with density proportional to the function $|x|^{\gamma}\left(1-x^{2}\right)^{\alpha-1 / 2}$ has the property (2.18). The result that there are no other measures with this property can be shown exactly in the same way as the corresponding statements in Theorems 2.1 and 2.2 .

The monic polynomials $\hat{S}_{n}^{(\alpha, \gamma)}(x)$ orthogonal with respect to the measure $|x|^{\gamma}\left(1-x^{2}\right)^{\alpha-1 / 2} d x$ can be obtained from [8, p. 156] as $\hat{S}_{-1}^{(\alpha, \gamma)}(x)=0, \hat{S}_{0}^{(\alpha, \gamma)}(x)=1$,

$$
\hat{S}_{n+1}^{(\alpha, \gamma)}(x)=x \hat{S}_{n}^{(\alpha, \gamma)}(x)-\gamma_{n+1}^{(\alpha, \gamma)} \hat{S}_{n-1}^{(\alpha, \gamma)}(x)
$$

where

$$
\gamma_{2 m}^{(\alpha, \gamma)}=\frac{(2 m-1+\gamma)(2 m-2+2 \alpha+\gamma)}{(4 m-4+2 \alpha+\gamma)(4 m-2+2 \alpha+\gamma)}
$$

$$
\gamma_{2 m+1}^{(\alpha, \gamma)}=\frac{2 m(2 m+2 \alpha-1)}{(4 m-2+2 \alpha+\gamma)(4 m+2 \alpha+\gamma)} .
$$

Consequently $\gamma_{n+1}^{(\alpha, \gamma)}=q_{2 n-2} p_{2 n}$ is a chain sequence with $\left(q_{0}=1\right)$

$$
p_{2 j}= \begin{cases}\frac{j}{2 \alpha+\gamma+2 j} & \text { if } j \text { is even, } \\ \frac{j+\gamma}{2 \alpha+\gamma+2 j} & \text { if } j \text { is odd . }\end{cases}
$$

Thus we have from (2.17), (2.20), (2.21) and straightforward calculations that

$$
\begin{aligned}
\Phi_{2 m}^{R^{*}}(z) & =\int_{-1}^{1} \frac{d \xi_{\alpha, \gamma, 2 m}^{R^{*}}(x)}{z-x}=\frac{1 \mid}{\mid z}-\frac{p_{4 m} \mid}{\mid z}-\frac{q_{4 m} p_{4 m-2} \mid}{\mid z}-\ldots-\frac{q_{4} p_{2} \mid}{\mid z} \\
& =\frac{1 \mid}{\mid z}-\frac{p_{4 m} \mid}{\mid z}-\frac{\gamma_{2 m}^{(\alpha+1, \gamma)} \mid}{\mid z}-\ldots-\frac{\gamma_{2}^{(\alpha+1, \gamma)} \mid}{\mid z} \\
& =\frac{\hat{S}_{2 m}^{(\alpha+1, \gamma)}(z)}{z \hat{S}_{2 m}^{(\alpha+1, \gamma)}(z)-p_{4 m} \hat{S}_{2 m-1}^{(\alpha+1, \gamma)}(z)} .
\end{aligned}
$$


Using the relations

$$
\begin{aligned}
& \hat{S}_{2 m}^{(\alpha+1, \gamma)}(z)=\frac{\Gamma(m+1) \Gamma\left(m+\alpha+1+\frac{\gamma}{2}\right)}{\Gamma\left(2 m+\alpha+1+\frac{\gamma}{2}\right)} P_{m}^{(\alpha+1 / 2,(\gamma-1) / 2)}\left(2 z^{2}-1\right), \\
& \hat{S}_{2 m-1}^{(\alpha+1, \gamma)}(z)=\frac{\Gamma(m) \Gamma\left(m+\alpha+1+\frac{\gamma}{2}\right)}{\Gamma\left(2 m+\alpha+\frac{\gamma}{2}\right)} z P_{m-1}^{(\alpha+1 / 2,(\gamma+1) / 2)}\left(2 z^{2}-1\right)
\end{aligned}
$$

(see [8, p. 156]), (2.21), formula (22.7.20) in Abramowitz and Stegun [1] it now follows that

$$
\begin{gathered}
z \hat{S}_{2 m}^{(\alpha+1, \gamma)}(z)-p_{4 m} \hat{S}_{2 m-1}^{(\alpha+1, \gamma)}(z) \\
=z \frac{\Gamma(m+1) \Gamma\left(m+\alpha+1+\frac{\gamma}{2}\right)}{\Gamma\left(2 m+\alpha+1+\frac{\gamma}{2}\right)}\left[P_{m}^{(\alpha+1 / 2,(\gamma-1) / 2)}\left(2 z^{2}-1\right)\right. \\
\left.-P_{m-1}^{(\alpha+1 / 2,(\gamma+1) / 2)}\left(2 z^{2}-1\right)\right] \\
=z \frac{\Gamma(m+1) \Gamma\left(m+\alpha+1+\frac{\gamma}{2}\right)}{\Gamma\left(2 m+\alpha+1+\frac{\gamma}{2}\right)} P_{m}^{(\alpha-1 / 2,(\gamma+1) / 2)}\left(2 z^{2}-1\right)
\end{gathered}
$$

and the Stieltjes transform of $\xi_{\alpha, \gamma, 2 m}^{R^{*}}$ is obtained as

$$
\Phi_{2 m}^{R^{*}}(z)=\frac{P_{m}^{(\alpha+1 / 2,(\gamma-1) / 2)}\left(2 z^{2}-1\right)}{z P_{m}^{(\alpha-1 / 2,(\gamma+1) / 2)}\left(2 z^{2}-1\right)} .
$$

Consequently the support points of $\xi_{\alpha, \gamma, 2 m}^{R^{*}}$ are given by the polynomial in the denominator of (2.23) and we have from Szegö [17, p. 63] for all nonvanishing support points $z_{0}$ of $\xi_{\alpha, \gamma, 2 m}^{R^{*}}$

$$
\begin{aligned}
\frac{d}{d z}( & \left.z P_{m}^{(\alpha-1 / 2,(\gamma+1) / 2)}\left(2 z^{2}-1\right)\right)\left.\right|_{z=z_{0}} \\
= & P_{m}^{(\alpha-1 / 2,(\gamma+1) / 2)}\left(2 z_{0}^{2}-1\right)+(2 m+2 \alpha+\gamma+2) z_{0}^{2} P_{m-1}^{(\alpha+1 / 2,(\gamma+3) / 2)}\left(2 z_{0}^{2}-1\right) \\
= & \frac{m+\alpha+\frac{\gamma}{2}+1}{m+\frac{\alpha+1}{2}+\frac{\gamma}{4}}\left[\left(m+\frac{\gamma+1}{2}\right) P_{m}^{(\alpha+1 / 2,(\gamma-1) / 2)}\left(2 z_{0}^{2}-1\right)\right. \\
& \left.-\left(m+\frac{\gamma+1}{2}\right) P_{m}^{(\alpha-1 / 2,(\gamma+1) / 2)}\left(2 z_{0}^{2}-1\right)+m P_{m}^{(\alpha+1 / 2,(\gamma+1) / 2)}\left(2 z_{0}^{2}-1\right)\right] \\
= & (2 m+\gamma+1) P_{m}^{(\alpha+1 / 2,(\gamma-1) / 2)}\left(2 z_{0}^{2}-1\right) .
\end{aligned}
$$

Here the second equality follows from the first by the formulas $(22.7 .16,22.7 .20)$ in [1] while the last equality follows from the second by formula $(22.7 .19,22.7 .20)$ (in the same reference) and $P_{m}^{(\alpha-1 / 2,(\gamma+1) / 2)}\left(2 z_{0}^{2}-1\right)=0$. Thus we obtain from $(2.23)$ for every nonvanishing support point $z_{0}$ of $\xi_{\alpha, \gamma, 2 m}^{R^{*}}$

$$
\xi_{\alpha, \gamma, 2 m}^{R^{*}}\left(z_{0}\right)=\frac{P_{m}^{(\alpha+1 / 2,(\gamma-1) / 2)}\left(2 z_{0}^{2}-1\right)}{\left.\frac{d}{d z}\left(P_{m}^{(\alpha-1 / 2,(\gamma+1) / 2)}\left(2 z^{2}-1\right)\right)\right|_{z=z_{0}}}=\frac{1}{2 m+\gamma+1}
$$

which proves the assertion of the theorem. 


\section{Generalized SIEVED ULTRASPHERICAL POLYNOMIALS}

In Theorem 2.3 of Section 2 we considered the 1 th, 3 th, 5 th, 7 th . . . convergent of the continued fraction expansion for the Stieltjes transform of a given probability measure on the interval $[-1,1]$, reversed the sequences of corresponding $p_{2 i}$ 's and determined all measures with the property(2.18). A natural extension of this proceeding is to terminate the continued fraction only at the positions $k, 3 k, 5 k$, $\ldots$ (for a given $k \in \mathbb{N}$ ) and investigate if there exist similar characterizations. We will show in this section that these problems are related to the so called "sieved random walk polynomials".

Following the work of [5], [6] and [12] we use a set of "random walk polynomials"

$$
\left\{\begin{array}{l}
x R_{n}(x)=A_{n} R_{n+1}(x)+B_{n} R_{n-1}(x), \quad n \geq 0, \\
R_{-1}(x)=0, R_{0}(x)=1
\end{array}\right.
$$

$\left(A_{n}, B_{n}>0, A_{n}+B_{n}=1, n \geq 0\right)$ in order to define "sieved random walk polynomials of the first kind" by

$$
\left\{\begin{array}{l}
x r_{n}(x)=b_{n-1} r_{n+1}(x)+a_{n-1} r_{n-1}(x), \quad n \geq 1 \\
r_{1}(x)=x, r_{0}(x)=1
\end{array}\right.
$$

and "sieved random walk polynomials of the second kind" by

$$
\left\{\begin{array}{l}
x s_{n}(x)=a_{n} s_{n+1}(x)+b_{n} s_{n-1}(x), \quad n \geq 1 \\
s_{1}(x)=2 x, s_{0}(x)=1
\end{array}\right.
$$

where

$$
a_{n}=b_{n}=\frac{1}{2} \text { if } k \nmid n+1, a_{n k-1}=A_{n-1}, \quad b_{n k-1}=B_{n-1}
$$

and $k \geq 2$ is a fixed integer. In the following we are interested in the polynomials $r_{n}(x)$ and $s_{n}(x)$ when one uses the generalized ultraspherical polynomials $C_{n}^{(\alpha+1, \gamma)}(x)$ defined in Section 2 as random walk polynomials. From [8, p. 156] we see that in this case

$$
A_{2 m}=1-B_{2 m}=\frac{2 m+1+\gamma}{4 m+2 \alpha+\gamma+2}
$$

$$
A_{2 m-1}=1-B_{2 m-1}=\frac{2 m}{4 m+2 \alpha+\gamma}
$$

(note that the $A_{n}$ 's correspond to the $p_{2 n}$ 's in $(2.21)$, i.e. $A_{n-1}=p_{2 n}$ ). Throughout this paper we will denote the polynomials obtained from (3.1), (3.2), (3.3), (3.4) and (3.5) as "generalized sieved ultraspherical polynomials of the first kind" $C_{n}^{(\alpha, \gamma)}(x, k)$ and "generalized sieved ultraspherical polynomials of the second kind"

$B_{n}^{(\alpha, \gamma)}(x, k)$. A straightforward calculation yields the recursive relations $C_{0}^{(\alpha, \gamma)}(x, k)$ 
$=1, C_{1}^{(\alpha, \gamma)}(x, k)=x$,

$$
\left\{\begin{array}{rr}
(2 \alpha+\gamma+2 n) x C_{n k}^{(\alpha, \gamma)}(x, k)=(2 \alpha+\gamma+n) C_{n k+1}^{(\alpha, \gamma)}(x, k)+n C_{n k-1}^{(\alpha, \gamma)}(x, k) & \\
& \text { if } n \text { is even } \\
(2 \alpha+\gamma+2 n) x C_{n k}^{(\alpha, \gamma)}(x, k)=(2 \alpha+n) C_{n k+1}^{(\alpha, \gamma)}(x, k)+(n+\gamma) C_{n k-1}^{(\alpha, \gamma)}(x, k) & \text { if } n \text { is odd } \\
2 x C_{j}^{(\alpha, \gamma)}(x, k)=C_{j+1}^{(\alpha, \gamma)}(x, k)+C_{j-1}^{(\alpha, \gamma)}(x, k) \quad & \text { if } j \neq n k
\end{array}\right.
$$

and $B_{0}^{(\alpha, \gamma)}(x, k)=1, B_{1}^{(\alpha, \gamma)}(x, k)=2 x$,

$$
\left\{\begin{array}{r}
(2 \alpha+\gamma+2 n) x B_{n k-1}^{(\alpha, \gamma)}(x, k)=n B_{n k}^{(\alpha, \gamma)}(x, k)+(2 \alpha+\gamma+n) B_{n k-2}^{(\alpha, \gamma)}(x, k) \\
\text { if } n \text { is even, } \\
(2 \alpha+\gamma+2 n) x B_{n k-1}^{(\alpha, \gamma)}(x, k)=(n+\gamma) B_{n k}^{(\alpha, \gamma)}(x, k)+(n+2 \alpha) B_{n k-2}^{(\alpha, \gamma)}(x, k) \\
\text { if } n \text { is odd } \\
2 x B_{j}^{(\alpha, \gamma)}(x, k)=B_{j+1}^{(\alpha, \gamma)}(x, k)+B_{j-1}^{(\alpha, \gamma)}(x, k) \text { if } j+1 \neq n k .
\end{array}\right.
$$

Observing (3.6) and (3.7) we see that these polynomials coincide with the sieved Jacobi polynomials introduced in [4] as a limit of the $q$-Wilson polynomials. Note also that the case $\gamma=0$ gives the sieved ultraspherical polynomials which were obtained in [2] as a limit from the $q$-ultraspherical polynomials of [16]. The corresponding measure of orthogonality can be obtained from [11, p. 561] and [12, p. 96] as

$$
w_{1}(x, \alpha, \gamma)=\left(1-x^{2}\right)^{\alpha-1 / 2}\left|U_{k-1}(x)\right|^{2 \alpha}\left|T_{k}(x)\right|^{\gamma} \quad(x \in[-1,1])
$$

for the generalized sieved ultraspherical polynomials of the first kind and as

$$
w_{2}(x, \alpha, \gamma)=\left(1-x^{2}\right)^{\alpha+1 / 2}\left|U_{k-1}(x)\right|^{2 \alpha}\left|T_{k}(x)\right|^{\gamma} \quad(x \in[-1,1])
$$

for the polynomials of the second kind. The following theorem characterizes the generalized sieved ultraspherical polynomials of the first kind by a similar "reversing property" as stated in Section 2.

Theorem 3.1. The generalized sieved ultraspherical polynomials of the first kind orthogonal with respect to the measure $d \xi_{\alpha, \gamma}(x)=w_{1}(x, \alpha, \gamma) d x$ defined by (3.6) can be characterized as the unique sieved random walk polynomials of the first kind whose corresponding probability measure $\mu$ satisfies for all $m \in \mathbb{N}_{0}$

$$
\left\{\begin{array}{l}
-\mu_{k(2 m+1)-1}^{R} \text { has equal masses at the zeros of } T_{k}(x) \\
-\mu_{k(2 m+1)-1}^{R} \text { has equal (but not necessary the same) masses at the } \\
\quad \text { remaining } 2 m k \text { support points. }
\end{array}\right.
$$

Moreover, for all $m \in \mathbb{N}$ the masses of $\xi_{\alpha, \gamma, k(2 m+1)-1}^{R}$ at the zeros of $T_{k}(z)$ are $\gamma+1$ times bigger than the (equal) masses of $\xi_{\alpha, \gamma, k(2 m+1)-1}^{R}$ at the remaining $2 m k$ support points. 
Proof. Consider a set of random walk polynomials defined by (3.1) and the corresponding set of sieved polynomials of the first kind in (3.2) orthogonal with respect to the measure $\mu$. Observing (3.2) and (3.4) it is straightforward to see that the minimal chain sequence $\left(q_{2 i-2} p_{2 i}\right)_{i \in \mathbb{N}}\left(q_{0}=1\right)$ corresponding to the monic orthogonal polynomials satisfies

$$
p_{2 i}=\frac{1}{2}, \quad i \neq n k,
$$

and we obtain from (2.15) for the Stieltjes transform of $\mu$

$$
\begin{aligned}
\Phi(z)= & \int_{-1}^{1} \frac{d \mu(x)}{z-x}=\frac{1 \mid}{\mid z}-\frac{p_{2} \mid}{\mid z}-\frac{q_{2} p_{4} \mid}{\mid z}-\frac{q_{4} p_{6} \mid}{\mid z}-\ldots \\
= & \frac{1 \mid}{\mid z}-\frac{\frac{1}{2} \mid}{\mid z}-\underbrace{\frac{\frac{1}{4} \mid}{\mid z}-\ldots-\frac{\frac{1}{4} \mid}{\mid z}}_{k-2}-\frac{\frac{1}{2} p_{2 k} \mid}{\mid z}-\frac{\frac{1}{2} q_{2 k} \mid}{\mid z} \\
& -\underbrace{\frac{\frac{1}{4} \mid}{\mid z}-\ldots-\frac{\frac{1}{4} \mid}{\mid z}}_{k-2}-\frac{\frac{1}{2} p_{4 k} \mid}{\mid z}-\frac{\frac{1}{2} q_{4 k} \mid}{\mid z}-\ldots
\end{aligned}
$$

A contraction such that the resulting continued fraction attains successively the values of the $(k-1)$ th, $(2 k-1)$ th, $(3 k-1)$ th $\ldots$ convergent yields (see $[15$, p. 12])

$$
\Phi(z)=\left(z-\frac{1}{2} H(z)\right)^{-1}
$$

where

$$
\begin{aligned}
H(z)= & 2 \frac{U_{k-2}(z)}{U_{k-1}(z)}+\frac{\left(\frac{1}{2}\right)^{2 k-3} p_{2 k} \mid}{\mid\left(\frac{1}{2}\right)^{2 k-2} U_{k-1}(z) T_{k}(z)}-\frac{\left(\frac{1}{2}\right)^{3 k-3} q_{2 k} p_{4 k} U_{k-1}(z) \mid}{\left(\frac{1}{2}\right)^{k-1} T_{k}(z)} \\
& -\frac{\left(\frac{1}{2}\right)^{2 k-2} q_{4 k} p_{6 k} \mid}{\mid\left(\frac{1}{2}\right)^{k-1} T_{k}(z)} \ldots \\
= & \frac{2}{U_{k-1}(z)}\left[U_{k-2}(z)+\frac{p_{2 k} \mid}{\mid T_{k}(z)}-\frac{q_{2 k} p_{4 k} \mid}{\mid T_{k}(z)}-\frac{q_{4 k} p_{6 k} \mid}{\mid T_{k}(z)}-\ldots\right] .
\end{aligned}
$$

Thus we obtain from (3.12)

$$
\begin{aligned}
\Phi(z) & =\int_{-1}^{1} \frac{d \mu(x)}{z-x} \\
& =U_{k-1}(z)\left[\frac{1}{\mid T_{k}(z)}-\frac{p_{2 k} \mid}{\mid T_{k}(z)}-\frac{q_{2 k} p_{4 k} \mid}{\mid T_{k}(z)}-\frac{q_{4 k} p_{6 k} \mid}{\mid T_{k}(z)}-\ldots\right] \\
& =U_{k-1}(z) \int_{-1}^{1} \frac{d \mu^{*}(x)}{T_{k}(z)-x}
\end{aligned}
$$

where $\mu^{*}$ is the probability measure defined by the recursive relation of the monic orthogonal polynomials $R_{-1}^{\prime}(x)=0, R_{0}^{\prime}(x)=1$

$$
\left\{\begin{aligned}
R_{n+1}^{\prime}(x) & =x R_{n}^{\prime}(x)-q_{2(n-1) k} p_{2 n k} R_{n-1}^{\prime}(x) \\
& =x R_{n}^{\prime}(x)-B_{n-2} A_{n-1} R_{n-1}^{\prime}(x)
\end{aligned}\right.
$$


$\left(q_{0}=1, A_{-1}=0\right)$, see also $[6$, p. 82$]$ or $[11$, p. 562$]$.

Now assume that $\xi_{\alpha, \gamma}$ is the probability measure with density proportional to the weight function (3.8) such that the generalized sieved ultraspherical polynomials $C_{n}^{(\alpha, \gamma)}(x, k)$ of the first kind are orthogonal with respect to the measure $d \xi_{\alpha, \gamma}(x)$. From (3.5) it follows that the polynomials $R_{l}^{\prime}(x)$ coincide with the polynomials $\hat{S}_{l}^{(\alpha, \gamma)}(x)$ in (2.19). Therefore the measure $\xi_{\alpha, \gamma}^{*}$ defined by (3.15) corresponds to the sequence $(2.21)$ and has density proportional to $|x|^{\gamma}\left(1-x^{2}\right)^{\alpha-1 / 2}$. A similar reasoning as used in the derivation of (3.13) gives

$$
\begin{aligned}
\Phi_{k(2 m+1)-1}^{R}(z) & =\int_{-1}^{1} \frac{d \xi_{\alpha, \gamma, k(2 m+1)-1}^{R}(x)}{z-x}=U_{k-1}(z) \int_{-1}^{1} \frac{d \xi_{\alpha, \gamma, 2 m}^{R^{*}}(x)}{T_{k}(z)-x} \\
& =: U_{k-1}(z) \Phi_{2 m}^{R^{*}}\left(T_{k}(z)\right)=U_{k-1}(z) \frac{V_{2 m}\left(T_{k}(z)\right)}{W_{2 m+1}\left(T_{k}(z)\right)}
\end{aligned}
$$

where $\xi_{\alpha, \gamma, 2 m}^{R^{*}}$ is defined in (2.23) and $V_{2 m}(z), W_{2 m+1}(z)$ are polynomials of degree $2 m$ and $2 m+1$, respectively. Consequently Theorem 2.3 yields for all $z_{0} \in$ $\operatorname{supp}\left(\xi_{\alpha, \gamma, k(2 m+1)-1}^{R}\right)$

$$
\begin{aligned}
\xi_{\alpha, \gamma, k(2 m+1)-1}^{R}\left(z_{0}\right) & =\left.\left(z-z_{0}\right) \Phi_{k(2 m+1)-1}^{R}(z)\right|_{z=z_{0}} \\
& =\left.\left(z-z_{0}\right) U_{k-1}\left(z_{0}\right) \Phi_{2 m}^{R^{*}}\left(T_{k}(z)\right)\right|_{z=z_{0}} \\
& =\frac{U_{k-1}\left(z_{0}\right) V_{2 m}\left(T_{k}\left(z_{0}\right)\right)}{\left.\frac{d}{d z} W_{2 m+1}\left(T_{k}(z)\right)\right|_{z=z_{0}}} \\
& =\frac{1}{k} \xi_{\alpha, \gamma, 2 m}^{R^{*}}\left(T_{k}\left(z_{0}\right)\right)= \begin{cases}\frac{1}{k} \frac{\gamma+1}{2 m+1+\gamma} & \text { if } T_{k}\left(z_{0}\right)=0, \\
\frac{1}{k} \frac{1}{2 m+1+\gamma} & \text { if } T_{k}\left(z_{0}\right) \neq 0\end{cases}
\end{aligned}
$$

which shows that $\xi_{\alpha, \gamma, k(2 m+1)-1}^{R}$ satisfies (3.10) for all $m \in \mathbb{N}_{0}$. On the other hand, if $\mu$ denotes a measure of orthogonality for a set of sieved random walk polynomials of the first kind satisfying (3.10), then similar arguments as given above show that the measure $\mu^{*}$ defined by (3.14) and (3.15) satisfies (2.13), which determines $\mu^{*}$ uniquely in the set of all symmetric probability measures on the interval $[-1,1]$ (by Theorem 2.3). Therefore (observing (3.11)) $\mu$ is unique and the assertion of the theorem follows.

The following result states an analogous characterization for the generalized sieved ultraspherical polynomials of the second kind. Its proof is performed by similar arguments as given in the proof of Theorem 3.1 and therefore omitted.

Theorem 3.2. The generalized sieved ultraspherical polynomials of the second kind orthogonal with respect to the measure $d \eta_{\alpha, \gamma}(x)=w_{2}(x, \alpha, \gamma) d x$ defined in (3.7) can be characterized as the unique sieved random walk polynomials of the second kind 
whose corresponding probability measure $\mu$ satisfies for all $m \in \mathbb{N}_{0}$

$$
\left\{\begin{array}{l}
-\mu_{k(2 m+2)-2}^{R} \text { has equal masses at the zeros of } T_{k}(z), \\
-\mu_{k(2 m+2)-2}^{R} \text { has equal masses at the zeros of } U_{k-1}(z), \\
-\mu_{k(2 m+2)-2}^{R} \text { has equal masses at the remaining } 2 m k \text { support points. }
\end{array}\right.
$$

Moreover, for all $m \in \mathbb{N}$ the masses of $\eta_{\alpha, \gamma, k(2 m+2)-2}^{R}$ at the zeros of $U_{k-1}(z)$ and $T_{k}(z)$ are $2 \alpha+1$ and $\gamma+1$ times bigger than the (equal) masses of $\eta_{\alpha, \gamma, k(2 m+2)-2}^{R}$ at the remaining $2 \mathrm{mk}$ support points.

Remark 3.3. It is worthwhile to mention that there exist a couple of similar properties of the weight functions (3.8), (3.9) of the generalized sieved ultraspherical polynomials of the first and second kind. For example, if $\xi_{\alpha, \gamma}$ is the probability measure with density proportional to (3.8), then it can be shown that for all $m \in \mathbb{N}$

$$
-\xi_{\alpha, \gamma, 2 m k-1}^{R} \text { has equal masses at all } 2 m k \text { support points. }
$$

Similarly it follows for the measure $\eta_{\gamma, \alpha}$ with density proportional to (3.9) that for all $m \in \mathbb{N}_{0}$

- $\eta_{\gamma, \alpha, k(2 m+1)-2}^{R}$ has equal masses $\frac{2 \alpha+1}{2 m k+(2 \alpha+1)(k-1)}$ at the zeros of $U_{k-1}(z)$,

- $\eta_{\gamma, \alpha, k(2 m+1)-2}^{R}$ has equal masses $\frac{1}{2 m k+(2 \alpha+1)(k-1)}$ at the remaining $2 m k$ support points.

Finally we remark that we conjecture that the properties (3.10) and (3.16) characterize the generalized sieved ultraspherical polynomials of the first and of the second kind in the class of all symmetric orthogonal polynomials on the interval $[-1,1]$.

\section{Asymptotic distribution of the zeros}

In this section we investigate the asymptotic behaviour of the zeros of the generalized sieved ultraspherical polynomials and generalized Hermite polynomials defined in Sections 3 and 2 when the degree and the parameters tend to infinity. To this end consider a set of random walk polynomials defined by (3.1) and (3.4) where the parameters $\alpha$ and $\gamma$ depend on the degree $n$, that is

$$
A_{j}^{(n)}=1-B_{j}^{(n)}=\left\{\begin{array}{ll}
\frac{j+1+\gamma_{n}}{2 \alpha_{n}+\gamma_{n}+2 j+2} & \text { if } j \text { is even, } \\
\frac{j+1}{2 \alpha_{n}+\gamma_{n}+2 j+2} & \text { if } j \text { is odd, }
\end{array} \quad j=0, \ldots, n-1,\right.
$$

and the corresponding sieved random walk polynomials $r_{l}(x)$ of the first kind where we use the set $\left\{A_{0}^{(n)}, A_{1}^{(n)}, \ldots, A_{n-1}^{(n)}\right\}$ in (3.2) if $n k+1 \leq l \leq(n+1) k$. Thus the parameters of the generalized sieved ultraspherical polynomials of the first kind depend on the degree and we obtain $r_{l}(x)=C_{l}^{\left(\alpha_{l}^{*}, \gamma_{l}^{*}\right)}(x, k)$ where

$$
\left\{\begin{array}{l}
\alpha_{l}^{*}=\alpha_{\lfloor(l-1) / k\rfloor} \\
\gamma_{l}^{*}=\gamma_{\lfloor(l-1) / k\rfloor}
\end{array}\right.
$$

and $\lfloor x\rfloor$ denotes the largest integer less than or equal to $x$. For $y \in[-1,1]$ let

$$
N_{l}^{\left(\alpha_{l}^{*}, \gamma_{l}^{*}\right)}(y):=\#\left\{x \leq y \mid C_{l}^{\left(\alpha_{l}^{*}, \gamma_{l}^{*}\right)}(x, k)=0\right\}
$$


denote the number of zeros of $C_{l}^{\left(\alpha_{l}^{*}, \gamma_{l}^{*}\right)}(x, k)$ less than or equal to $y$. In order to discuss the asymptotic behaviour of $N_{l}^{\left(\alpha_{l}^{*}, \gamma_{l}^{*}\right)}$ when $l \rightarrow \infty, \alpha_{l} \rightarrow \infty, \gamma_{l} \rightarrow \infty$ we need the following auxiliary result.

Lemma 4.1. Let $\rho$ denote a symmetric probability measure on the interval $[-1,1]$ with Stieltjes transform

$$
\Phi^{*}(z)=\int_{-1}^{1} \frac{d \rho(x)}{z-x}=\frac{1 \mid}{\mid z}-\frac{p_{2} \mid}{\mid z}-\frac{q_{2} p_{4} \mid}{\mid z}-\frac{q_{4} p_{6} \mid}{\mid z}-\ldots
$$

such that for some $k \in \mathbb{N}, g, h \in(0,1)$

$$
p_{2 l}= \begin{cases}g & \text { if } l=k j \text { and } j \text { is odd } \\ h & \text { if } l=k j \text { and } j \text { is even, } \\ \frac{1}{2} & \text { else, }\end{cases}
$$

then

$$
\Phi^{*}(z)=\frac{1}{2 h} \frac{(1-2 h) T_{k}^{2}(z)+(h-g)-\sqrt{\left(T_{k}^{2}(z)-\kappa\right)^{2}-4 \mu}}{U_{k-1}(z) T_{k}(z)\left(1-z^{2}\right)}
$$

where

$$
\left\{\begin{array}{l}
\kappa=g(1-h)+h(1-g), \\
\mu=g(1-g) h(1-h)
\end{array}\right.
$$

and the sign of the square root is defined such that

$$
\left|\frac{T_{k}^{2}(z)-\kappa}{2 \sqrt{\mu}}+\sqrt{\frac{\left(T_{k}^{2}(z)-\kappa\right)^{2}}{4 \mu}-1}\right|>1 .
$$

Proof. Similarly as in the first part of the proof of Theorem 3.1 a contraction such that the convergent of the resulting continued fraction attains successively the values of the $(k-1)$ th, $(2 k-1)$ th, $(3 k-1)$ th, $\ldots$ convergent, yields

$$
\Phi^{*}(z)=\left[z-\frac{1}{2} \Psi(z)\right]^{-1}
$$

where

$$
\begin{aligned}
& \Psi(z)=\frac{1 \mid}{\mid z}-\underbrace{\frac{\frac{1}{4} \mid}{\mid z}-\ldots-\frac{\frac{1}{4} \mid}{\mid z}}_{k-2}-\frac{\frac{1}{2} g \mid}{\mid z}-\frac{\frac{1}{2}(1-g) \mid}{\mid z} \\
&-\underbrace{\frac{\frac{1}{4} \mid}{\mid z}-\ldots-\frac{\frac{1}{4} \mid}{\mid z}}_{k-2}-\frac{\frac{1}{2} h \mid}{\mid z}-\frac{\frac{1}{2}(1-h) \mid}{\mid z}-\frac{\frac{1}{4} \mid}{\mid z}-\ldots \\
&=\frac{2}{U_{k-1}(z)}\left[U_{k-2}(z)+\frac{g \mid}{\mid T_{k}(z)}-\frac{h(1-g) \mid}{\mid T_{k}(z)}-\frac{g(1-h) \mid}{\mid T_{k}(z)}\right. \\
&\left.-\frac{h(1-g) \mid}{\mid T_{k}(z)}-\ldots\right] .
\end{aligned} .
$$


By (4.7) and a further even contraction it now follows that

$$
\begin{aligned}
& \Psi(z)=U_{k-1}(z) T_{k}(z)\left[\frac{1}{\mid T_{k}^{2}(z)-g}-\frac{g(1-g) h \mid}{\mid T_{k}^{2}(z)-\kappa}-\frac{\mu}{\mid T_{k}^{2}(z)-\kappa}\right. \\
& \left.-\frac{\mu}{\mid T_{k}^{2}(z)-\kappa}-\ldots\right]
\end{aligned}
$$

where $\mu$ and $\kappa$ are defined in (4.5). The assertion is now a consequence from standard results noting that the distribution with density $\frac{2}{\pi} \sqrt{1-x^{2}}(x \in[-1,1])$ has the Stieltjes transform

$$
\frac{2}{\pi} \int_{-1}^{1} \frac{\sqrt{1-x^{2}}}{w-x} d x=\frac{1 \mid}{\mid w}-\frac{\frac{1}{4} \mid}{\mid w}-\frac{\frac{1}{4} \mid}{\mid w}-\ldots=2\left(w-\sqrt{w^{2}-1}\right)
$$

where the square root is such that $\left|w+\sqrt{w^{2}-1}\right|>1$ (see e.g. VanAssche [18, p. 176]).

Remark 4.2. Note that the case $g=h$ was already discussed in [12] where sieved orthogonal polynomials on several intervals are considered (using the notation $g=$ $\left.h=\frac{1}{c+1}\right)$. By Lemma 5.1 in [6] and a delicate analysis depending on (4.6) we obtain that the function in (4.4) has no poles at the zeros of the polynomial $\left(1-z^{2}\right) U_{k-1}(z)$ provided that $g+h \leq 1$. If $g+h>1$ there are simple poles at \pm 1 with residues $\frac{g+h-1}{2 h k}$ and simple poles at the zeros of $U_{k-1}(x)$ with residues $\frac{g+h-1}{h k}$. Moreover, by Lemma 5.1 in [6] it can also be shown that $\Phi^{*}(z)$ has no poles at the zeros of $T_{k}(z)$ if $h \leq g$ and simple poles with residues $\frac{h-g}{h k}$ if $h>g$. Finally we mention that by the Perron-Stieltjes inversion formula the absolute continuous component of $d \rho(x)$ is supported on the set

$$
\overline{E_{k}(\kappa, \mu)}:=\overline{\left\{x \in[-1,1]|| T_{k}^{2}(x)-\left.\kappa\right|^{2}<4 \mu\right\}}
$$

with density

$$
\frac{d \rho(x)}{d x}=\frac{1}{2 \pi h} \frac{\sqrt{4 \mu-\left(T_{k}^{2}(x)-\kappa\right)^{2}}}{\left|U_{k-1}(x)\right|\left|T_{k}(x)\right|\left(1-x^{2}\right)} I\left\{x \in \overline{E_{k}(\kappa, \mu)}\right\}
$$

where $\kappa$ and $\mu$ are defined in (4.5).

Theorem 4.3. Let $\lim _{l \rightarrow \infty} \frac{\alpha_{l}}{l}=a \geq 0$ and $\lim _{l \rightarrow \infty} \frac{\gamma_{l}}{l}=c \geq 0$, then $N_{l}^{\left(\alpha_{l}^{*}, \gamma_{l}^{*}\right)}$ defined by (4.3) and (4.2) satisfies

$$
\begin{aligned}
\lim _{l \rightarrow \infty} \frac{1}{l} N_{l}^{\left(\alpha_{l}^{*}, \gamma_{l}^{*}\right)}(y) \\
\quad=\frac{2 a+c+2}{2 \pi} \int_{-1}^{y} \frac{\sqrt{4 \mu_{a, c}-\left(T_{k}^{2}(x)-\kappa_{a, c}\right)^{2}}}{\left|U_{k-1}(x)\right|\left|T_{k}(x)\right|\left(1-x^{2}\right)} I\left\{x \in \overline{E_{k}\left(\kappa_{a, c}, \mu_{a, c}\right)}\right\} d x
\end{aligned}
$$

with

$$
\begin{aligned}
\mu_{a, c} & =\frac{(2 a+1)(c+1)(2 a+c+1)}{(2 a+c+2)^{4}}, \\
\kappa_{a, c} & =\frac{(2 a+1)(c+2)+c(c+1)}{(2 a+c+2)^{2}} .
\end{aligned}
$$


Proof. Because $\alpha_{l}^{*}=\alpha_{\lfloor(l-1) / k\rfloor}, \gamma_{l}^{*}=\gamma_{\lfloor(l-1) / k\rfloor}$ are independent of $l \in\{n k+$ $1, \ldots,(n+1) k\}$, the zeros of

$$
C_{n k+1}^{\left(\alpha_{n k+1}^{*}, \gamma_{n k+1}^{*}\right)}(x, k), C_{n k+2}^{\left(\alpha_{n k+2}^{*}, \gamma_{n k+2}^{*}\right)}(x, k), \ldots, C_{(n+1) k}^{\left(\alpha_{(n+1) k}^{*}, \gamma_{(n+1) k}^{*}\right)}(x, k)
$$

are interlacing and consequently it is sufficient to prove (4.9) for the subsequence $l=n k$. The polynomial $C_{n k}^{\left(\alpha_{n k}^{*}, \gamma_{n k}^{*}\right)}(x, k)$ is proportional to the polynomial in the denominator of the Stieltjes transform of the probability measure $\xi_{\alpha_{n k}^{*}, \gamma_{n k}^{*}, n k-1}^{R}$ which is obtained by terminating the continued fraction expansion for the Stieltjes transform of $\xi_{\alpha_{n k}^{*}, \gamma_{n k}^{*}}$ with density (3.8) and reversing the corresponding sequence of $p_{2 i}$ 's (see (2.16) and (2.17)). By the results of Section $3 \xi_{\alpha_{n k}^{*}, \gamma_{n k}^{*}, n k-1}^{R}$ is "nearly" a uniform distribution on the set $\left\{x \mid C_{n k}^{\left(\alpha_{n k}^{*}, \gamma_{n k}^{*}\right)}(x, k)=0\right\}$ and we have to distinguish the following two cases:

(A) $n=2 m, l=2 m k$ : In this case it follows from Remark 3.3 that $\xi_{\alpha_{2 m k}^{*},}^{R}, \gamma_{2 m k}^{*}, 2 m k-1$ has equal masses at the zeros of $C_{2 m k}^{\left(\alpha_{2 m k}^{*}, \gamma_{2 m k}^{*}\right)}(x, k)$ and the $p_{2 i}^{(2 m)}$ in the corresponding continued fraction expansion

$$
\int_{-1}^{1} \frac{d \xi_{\alpha_{2 m k}^{*}, \gamma_{2 m k}^{*}, 2 m k-1}^{R}(x)}{z-x}=\frac{1 \mid}{\mid z}-\frac{p_{2}^{(2 m)} \mid}{\mid z}-\frac{q_{2}^{(2 m)} p_{4}^{(2 m)} \mid}{\mid z}-\ldots-\frac{q_{4 m k-4}^{(2 m)} p_{4 m k-2}^{(2 m)} \mid}{z} \ldots
$$

are given by $p_{2 j}^{(2 m)}=p_{2(2 m k-j)}(j=1, \ldots, 2 m k-1)$. From $p_{2 k j}=A_{j-1}^{(2 m-1)}$ $(j=1, \ldots, 2 m-1),(4.1)$, and (3.11) we have

$$
p_{2 k j}^{(2 m)}= \begin{cases}\frac{\gamma_{2 m-1}+2 m-j}{2 \alpha_{2 m-1}+\gamma_{2 m-1}+2(2 m-j)} & \text { if } j \text { is odd, } \\ \frac{2 m-j}{2 \alpha_{2 m-1}+\gamma_{2 m-1}+2(2 m-j)} & \text { if } j \text { is even }\end{cases}
$$

$(j \leq 2 m-1)$ and $p_{2 l}^{(2 m)}=\frac{1}{2}$ whenever $l \neq j k(l \leq 2 m k-1)$. From the assumption of Theorem 4.3 it follows that

$$
\lim _{m \rightarrow \infty} p_{2 l}^{(2 m)}=p_{2 l}^{*}= \begin{cases}\frac{1}{2} & \text { if } l \neq j k, \\ \frac{c+1}{2 a+c+2} & \text { if } l=j k, j \text { is odd } \\ \frac{1}{2 a+c+2} & \text { if } l=j k, j \text { is even }\end{cases}
$$

and by the same reasoning as in [9] we obtain that the uniform distribution on the set $\left\{x \mid C_{2 m k}^{\left(\alpha_{2 m k}^{*}, \gamma_{2 m k}^{*}\right)}(x, k)=0\right\}$ converges weakly to the distribution $\xi^{*}$ corresponding to the sequence $\left(p_{2}^{*}, p_{4}^{*}, p_{6}^{*}, \ldots\right)$ in (4.11). An application of Lemma 4.1 and Remark 4.2 shows that $\xi^{*}$ is absolute continuous with density given in (4.8) where

$$
g=\frac{c+1}{2 a+c+2}, h=\frac{1}{2 a+c+2}, \quad g+h \leq 1, h \leq g .
$$

This proves the assertion for the subsequence $l=2 m k$.

(B) $n=2 m+1, l=(2 m+1) k$ : By a similar argument as in part (4) we obtain that the probability measure $\xi_{\alpha_{(2 m+1) k}^{*}, \gamma_{(2 m+1) k}^{*},(2 m+1) k-1}^{R}$ converges weakly to the 
distribution $\xi^{* *}$ corresponding to the sequence $\left(p_{2}^{* *}, p_{4}^{* *}, p_{6}^{* *}, \ldots\right)$ where

$$
p_{2 l}^{* *}= \begin{cases}\frac{1}{2} & \text { if } l \neq j k, \\ \frac{1}{2 a+c+2} & \text { if } l=j k, j \text { is odd, } \\ \frac{c+1}{2 a+c+2} & \text { if } l=j k, j \text { is even. }\end{cases}
$$

By Remark 4.2 (with $g=\frac{1}{2 a+c+2}, h=\frac{c+1}{2 a+c+2}, g+h \leq 1, h \geq g$ ) the absolute continuous component of $\xi^{* *}$ has the density

$$
f_{a, c}(x)=\frac{2 a+c+2}{2 \pi(c+1)} \frac{\sqrt{4 \mu_{a, c}-\left(T_{k}^{2}(x)-\kappa_{a, c}\right)^{2}}}{\left|U_{k-1}(x)\right|\left|T_{k}(x)\right|\left(1-x^{2}\right)} I\left\{x \in \overline{E_{k}\left(\kappa_{a, c}, \mu_{a, c}\right)}\right\}
$$

where $\kappa_{a, c}, \mu_{a, c}$ are defined in (4.10) (note that $\kappa$ and $\mu$ defined by (4.5) are symmetric in $g$ and $h$ ). Moreover, there also exists a discrete part of $\xi^{* *}$ at the zeros of $T_{k}(z)$ with equal masses

$$
\frac{h-g}{k h}=\frac{c}{k(c+1)}
$$

(see Remark 4.2). Now Theorem 3.1 (and its proof) show that

$$
\xi_{\alpha_{(2 m+1) k}^{*}, \gamma_{(2 m+1) k}^{*},(2 m+1) k-1}^{R}
$$

has equal masses

$$
\frac{\gamma_{2 m}+1}{\left(2 m+\gamma_{2 m}+1\right) k}
$$

at the zeros of the Chebyshev polynomial $T_{k}(x)$ and masses

$$
\frac{1}{\left(2 m+\gamma_{2 m}+1\right) k}
$$

at the remaining $2 m k$ support points. This yields

$$
\begin{aligned}
& \frac{1}{(2 m+1) k} N_{(2 m+1) k}^{\left(\alpha_{(2 m+1) k}^{*}, \gamma_{(2 m+1) k}^{*}\right)}(y) \\
& =\frac{\left(2 m+\gamma_{2 m}+1\right) k}{(2 m+1) k}\left[\frac{\gamma_{2 m}+1}{\left(2 m+\gamma_{2 m}+1\right) k} \#\left\{x \leq y \mid T_{k}(x)=0\right\}\right. \\
& +\frac{1}{\left(2 m+\gamma_{2 m}+1\right) k} \#\left\{x \leq y \mid C_{(2 m+1) k}^{\left(\alpha_{(2 m+1) k}^{*}, \gamma_{(2 m+1) k}^{*}\right)}(x, k)=0,\right. \\
& \left.-\frac{\gamma_{2 m}}{\left(2 m+\gamma_{2 m}+1\right) k} \#\left\{x \leq y \mid T_{k}(x)=0\right\}\right] \\
& =\frac{\left(2 m+\gamma_{2 m}+1\right)}{(2 m+1)}\left[\int_{-1}^{y} d \xi_{\alpha_{(2 m+1) k}^{*}, \gamma_{(2 m+1) k}^{*},(2 m+1) k-1}^{R}(x)\right. \\
& \left.-\frac{\gamma_{2 m}}{\left(2 m+\gamma_{2 m}+1\right) k} \#\left\{x \leq y \mid T_{k}(x)=0\right\}\right],
\end{aligned}
$$


and in the limit

$$
\begin{aligned}
& \lim _{(2 m+1) k \rightarrow \infty} \frac{1}{(2 m+1) k} N_{(2 m+1) k}^{\left(\alpha_{(2 m+1) k}^{*}, \gamma_{(2 m+1) k}^{*}\right)}(y) \\
& =(c+1)\left[\int_{-1}^{y} d \xi^{* *}(x)-\frac{c}{(c+1) k} \#\left\{x \leq y \mid T_{k}(x)=0\right\}\right] \\
& =\frac{2 a+c+2}{2 \pi} \int_{-1}^{y} \frac{\sqrt{4 \mu_{a, c}-\left(T_{k}^{2}(x)-\kappa_{a, c}\right)^{2}}}{\left|U_{k-1}(x)\right|\left|T_{k}(x)\right|\left(1-x^{2}\right)} I\left\{x \in \overline{E_{k}\left(\kappa_{a, c}, \mu_{a, c}\right)}\right\} d x .
\end{aligned}
$$

This proves (4.9) for the subsequence $l=(2 m+1) k$ and by part (A) for the sequence $l=n k$. The assertion of the theorem follows from the discussion at the beginning of this proof.

The following result states the analogue for the asymptotic distribution of the zeros of the generalized sieved ultraspherical polynomials of the second kind and can be proved by similar arguments as given in the proof of Theorem 4.3.

Theorem 4.4. For $y \in[-1,1]$ let

$$
M_{l}^{\left(\alpha_{l}^{*}, \gamma_{l}^{*}\right)}(y):=\#\left\{x \leq y \mid B_{l}^{\left(\alpha_{l}^{*}, \gamma_{l}^{*}\right)}(x, k)=0\right\}
$$

denote the number of zeros of $B_{l}^{\left(\alpha_{l}^{*}, \gamma_{l}^{*}\right)}(x, k)$ less than or equal to $y$. If $\lim _{l \rightarrow \infty} \frac{\alpha_{l}}{l}$ $=a \geq 0$ and $\lim _{l \rightarrow \infty} \frac{\gamma_{l}}{l}=c \geq 0$, then

$$
\begin{aligned}
\lim _{l \rightarrow \infty} \frac{1}{l} M_{l}^{\left(\alpha_{l}^{*}, \gamma_{l}^{*}\right)}(y) \\
\quad=\frac{2 a+c+2}{2 \pi} \int_{-1}^{y} \frac{\sqrt{4 \mu_{a, c}-\left(T_{k}^{2}(x)-\kappa_{a, c}\right)^{2}}}{\left|U_{k-1}(x)\right|\left|T_{k}(x)\right|\left(1-x^{2}\right)} I\left\{x \in \overline{E_{k}\left(\kappa_{a, c}, \mu_{a, c}\right)}\right\} d x
\end{aligned}
$$

where $\mu_{a, c}, \kappa_{a, c}$ are defined in (4.10).

Note that in the case $c=0$ the limit distribution in Theorems 4.3 and 4.4 is exactly the distribution for which the sieved Chebyshev polynomials of the first kind (introduced in [12, p. 99] are orthogonal with respect to. In the case considered here the discrete spectrum of this measure is empty (the parameter $c$ in the last named reference corresponds to $2 a+1$ which is obviously $\geq 1$ (see [12, p. 99]). The case $a=c=0$ and the trigonometric identity yield the arcsin measure as limit distribution.

It is also worthwhile to mention that an alternative proof of the asymptotic results for zeros of random walk polynomials $r_{n k}(x)=R_{n}\left(T_{k}(x)\right)-R_{n-2}\left(T_{k}(x)\right.$ ) [or of the second kind $\left.s_{n k+k-1}(x)=U_{k-1}(x) R_{n}\left(T_{k}(x)\right)\right]$ could be obtained if the asymptotic distribution of the zeros of the polynomials $R_{n}(x)-R_{n-2}(x)$ [or $R_{n}(x)$ ] is known. This proof requires additionally a careful inspection of the inverse branches of $T_{k}(x)$ (see also [11]).

We will conclude this paper by discussing the corresponding limit statement for the zeros of the generalized Hermite polynomials $H_{n}^{(\gamma)}(x)$ defined in Section 2. As pointed out in the introduction this result has already been proved in [10] as an application of strong asymptotics for the Laguerre polynomials $L_{n}^{(\alpha)}(x)$ and the relation (1) between these and the generalized Hermite polynomials. The proof given here is based on the characterizing property (2.4) of the generalized Hermite polynomials (note that parametrization and standardization in [10] is different). 
Theorem 4.5. For $y \in \mathbb{R}$ let

$$
N_{l}^{\left(\gamma_{l}\right)}(y)=\#\left\{x \leq y \mid H_{l}^{\left(\gamma_{l}\right)}(x)=0\right\}
$$

denote the number of zeros of $H_{l}^{\left(\gamma_{l}\right)}(x)$ less than or equal to $y$. If $\lim _{l \rightarrow \infty} \frac{\gamma_{l}}{l}=$ $c \geq 0$, then

$$
\lim _{l \rightarrow \infty} \frac{1}{l} N_{l}^{\left(\gamma_{l}\right)}(\sqrt{l} y)=\frac{1}{\pi} \int_{-\infty}^{\xi} \frac{\sqrt{\left.(c+1)-\mid x^{2}-(c / 2)-1\right)\left.\right|^{2}}}{|x|} I\left\{x \in \overline{E_{1}}\right\} d x
$$

where $\bar{E}_{1}=\left\{x \in \mathbb{R}|| x^{2}-(c / 2)-1 \mid<\sqrt{c+1}\right\}$.

Proof. We only consider the case of an even subsequence $l=2 m$, the odd case is treated exactly in the same way as part (B) in the proof of Theorem 4.3. Let $\xi_{\gamma_{l}}$ denote the probability measure with density proportional to $|x|^{\gamma_{l}} \exp \left(-x^{2}\right)$ and let $\mu_{2 m-1}$ denote the uniform distribution on the set

$$
\left\{\frac{x}{\sqrt{2 m}} \mid H_{2 m}^{\left(\gamma_{2 m}\right)}(x)=0\right\} .
$$

From (2.4), (2.7) and (2.12) we obtain for the Stieltjes transform of $\mu_{2 m-1}$

$$
\int_{-\infty}^{\infty} \frac{d \mu_{2 m-1}(x)}{z-x}=\int_{-\infty}^{\infty} \frac{d \xi_{\gamma, 2 m-1}^{R}(x)}{z-x / \sqrt{2 m}}=\frac{1 \mid}{\mid z}-\frac{\tilde{\alpha}_{1}^{(2 m)} \mid}{\mid z}-\ldots-\frac{\tilde{\alpha}_{2 m-1}^{(2 m)} \mid}{\mid z}
$$

where

$$
\tilde{\alpha}_{j}^{(2 m)}= \begin{cases}\frac{1}{2} \frac{2 m-j}{2 m} & \text { if } j \text { is even, } \\ \frac{1}{2} \frac{2 m-j+\gamma_{2 m}}{2 m} & \text { if } j \text { is odd. }\end{cases}
$$

Thus we have

$$
\lim _{m \rightarrow \infty} \tilde{\alpha}_{j}^{(2 m)}= \begin{cases}\frac{1}{2}=: h & \text { if } j \text { is even, } \\ \frac{1}{2}(c+1)=: g & \text { if } j \text { is odd }\end{cases}
$$

and the same arguments as in [9] show that $\mu_{2 m-1}$ converges weakly to the distribution $\mu^{*}$ with Stieltjes transform

$$
\begin{aligned}
\Phi^{*}(z) & =\frac{1 \mid}{\mid z}-\frac{g \mid}{\mid z}-\frac{h \mid}{\mid z}-\frac{g \mid}{\mid z}-\frac{h \mid}{\mid z}-\ldots \\
& =\frac{z \mid}{\mid z^{2}-g}-\frac{g h \mid}{\mid z^{2}-(g+h)}-\frac{g h \mid}{\mid z^{2}-(g+h)}-\frac{g h \mid}{\mid z^{2}-(g+h)}-\ldots \\
& =\frac{z^{2}+h-g-\sqrt{\left[z^{2}-(g+h)\right]^{2}-4 h g}}{2 h z} .
\end{aligned}
$$

Here the square root is defined such that

$$
\left|\frac{z^{2}-(g+h)}{2 \sqrt{g h}}+\sqrt{\frac{\left[z^{2}-(g+h)\right]^{2}}{4 g h}-1}\right|>1
$$


and the second equality follows from the first by an even contraction while the second equality implies the third by the same arguments as given in the proof of Lemma 4.1. An application of (4.13) (which implies $h \leq g$ ) and the Perron-Stieltjes inversion formula show that $\mu^{*}$ is absolute continuous with the density in (4.12). This proves the assertion of the theorem.

\section{ACKNOWLEDGEMENTS}

Parts of this paper were written while the author was visiting the University of Göttingen. The author would like to thank the Institut für Mathematische Stochastik for its hospitality, Dick Askey and Walter VanAssche for their help with the references and an unknown referee for his constructive comments.

\section{REFERENCES}

1. M. Abramowitz and I. Stegun. Handbook of mathematical functions, Dover, New York, 1964.

2. W. Al-Salam, W. R. Allaway and R. Askey, Sieved ultraspherical polynomials, Trans. Amer. Math. Soc. 284 (1984), 39-55. MR 85j:33005

3. W. Al-Salam, Characterization theorems for orthogonal polynomials, Orthogonal Polynomials: Theory and Practice (P. Nevai, ed.), NATO ASI Series C 294, Kluwer, Dordrecht, 1990, pp. 1-24. MR 92g:42011

4. R. Askey, Orthogonal polynomials old and new, and some combinatorial connections, Enumeration and Design (D. M. Jackson and S. A. Vanstone, eds.), Academic Press, New York, 1984, pp. 67-84. MR 87a:05022

5. J. A. Charris and M. E. H. Ismail, On sieved orthogonal polynomials II: Random walk polynomials, Canad. J. Math. 38 (1986), 397-415. MR 87j:33014a

6. Math. Soc. 340 (1993), 71-93. MR 94a:33007

7. J. A. Charris, M. E. H. Ismail and S. Monsalve, On sieved orthogonal polynomials X: General blocks of recurrence relations, Pacific. J. Math. 163 (1994), 237-267. MR 95d:33004

8. T. S. Chihara, Introduction to orthogonal polynomials, Gordon \& Breach, New York, 1978. MR 58:1979

9. H. Dette and W. J. Studden, On a new characterization of the classical orthogonal polynomials, J. Approx. Theory 71 (1992), 3-17. MR 93k:33005

10. W. Gawronski, Strong asymptotics and asymptotic zero distribution of Laguerre polynomials $L_{n}^{(a n+\alpha)}(x)$ and Hermite polynomials $H_{n}^{(a n+\alpha)}(x)$, Analysis 13 (1993), 29-68. MR 95d:30010

11. J. S. Geronimo and W. VanAssche, Orthogonal polynomials on several intervals via a polynomial mapping, Trans. Amer. Math. Soc. 308 (1988), 559-581. MR 89f:42021

12. M. E. H. Ismail, On sieved orthogonal polynomials III: Orthogonality on several intervals, Trans. Amer. Math. Soc. 294 (1986), 89-111. MR 87j:33014b

13. S. Karlin and W. J. Studden, Tchebyscheff systems with applications in analysis and statistics, Interscience, New York, 1966. MR 34:4757

14. T. S. Lau and W. J. Studden, On an extremal problem of Fejér, J. Approx. Theory $\mathbf{5 3}$ (1988), 184-194. MR 89h:41007

15. O. Perron, Die Lehre von den Kettenbrüchen (Band I and II), Teubner, Stuttgart, 1954; 1957. MR 16:239e; MR 19:25c

16. L. J. Rogers, Third memoir on the expansion of certain infinite products, Proc. London Math. Soc. 26 (1895), 15-32.

17. G. Szegö, Orthogonal polynomials, Amer. Math. Soc. Colloq. Publ., vol. 23, Providence, RI, 1975. MR 51:8724

18. W. VanAssche, Asymptotics for orthogonal polynomials, Lecture Notes in Math., vol. 1265, Springer-Verlag, Berlin and New York, 1987. MR 88i:42035

19. H. S. Wall, Analytic theory of continued fractions, Van Nostrand, New York, 1948. MR 10:33a

Institut für Mathematische Stochastik, Technische Universität Dresden, MommsenStr. 13, 01062 Dresden, Germany

E-mail address: dette@math.tu-dresden.de 